\title{
ㄷำ1
}

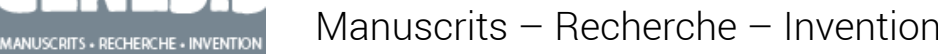

43 | 2016

Bande dessinée

\section{Bande dessinée : une génétique à ciel ouvert}

\section{Luc Vigier}

\section{OpenEdition}

\section{Journals}

Édition électronique

URL : http://journals.openedition.org/genesis/1704

DOI : 10.4000/genesis. 1704

ISSN : 2268-1590

\section{Éditeur :}

Presses universitaires de Paris Sorbonne (PUPS), Société internationale de génétique artistique littéraire et scientifique (SIGALES)

\section{Édition imprimée}

Date de publication : 12 décembre 2016

Pagination : 51-63

ISBN : 9791023105490

ISSN : 1167-5101

\section{Référence électronique}

Luc Vigier, «Bande dessinée : une génétique à ciel ouvert », Genesis [En ligne], 43 | 2016, mis en ligne le 28 novembre 2017, consulté le 21 avril 2019. URL : http://journals.openedition.org/genesis/1704 ;

DOI : 10.4000/genesis. 1704 


\title{
Bande dessinée : une génétique à ciel ouvert
}

\author{
Luc Vigier
}

$\mathrm{L}$

e processus de légitimation de la bande dessinée comme art et comme littérature tout au long de la seconde moitié du XXe siècle s'est accompagné d'une médiatisation de plus en plus large de ses secrets de fabrication. Dans un contexte éditorial aujourd'hui florissant ${ }^{1}$ et un univers médiatique étendu, la bande dessinée affiche désormais sans complexe, comme aucune autre forme d'art à l'exception du cinéma, ses coulisses techniques : expositions d'originaux, ventes de planches ${ }^{2}$, mais aussi images d'artistes au travail, visites d'ateliers. L'explication des contraintes, des codes, des règles, des procédures et des étapes prolifère également, notamment sur le web et les réseaux, sous forme de reportages pédagogiques ou expérimentaux de toutes sortes, voulus par des spécialistes de bande dessinée ou financés par les éditeurs qui ont compris et assumé la puissance visuelle du teasing graphique, calquée sur celle du marketing filmique. Cet ensemble constitue de fait un fonds nouveau mais problématique d'informations sur la pratique de la bande dessinée : chantier à ciel ouvert, la génétique de la bande dessinée, présente aujourd'hui dans les espaces éditoriaux les plus variés, relève autant de l'archéologie que du spectacle.

Les lieux d'exposition des croquis, des roughs, des étapes, des storyboards ou des documents préparatoires ont d'abord été les albums eux-mêmes, qui dans les années soixante-dix commencent à proposer des dossiers de brouillons, des exemples de croquis 3 , sur le modèle littéraire des manuscrits ajoutés à l'édition, comme cela se faisait pour certains tirages de tête au XIXe siècle et au début du XXe. La valorisation du travail préparatoire s'exerce alors contre l'idée reçue d'une facilité des dessins destinés à un pur divertissement. Simultanément, elle crée un intervalle difficile à définir - et parfois un abîme - entre le plaisir de l'inachevé et la finition impeccable de l'album, qui fixe les formes. L'insertion d'esquisses, de pages de carnets ou de brouillons de scénarios établit aux marges de l'album des faits instables et mouvants qui semblent contrarier la cristallisation éditoriale : ils font presque vivre sur les côtés une autre œuvre qui vient constituer une strate virtuelle ajoutée de la lecture, cachée sous l'image éditée. L'autre lieu est ensuite l'exposition en festival (Quai des Bulles à Saint-Malo, Festival d'Angoulême) mais aussi désormais dans les musées.

Sur les murs sont affichés les dessins originaux, dans des dispositifs identiques à ceux des œuvres de grands peintres, graveurs ou sculpteurs et l'on regarde, sidéré, la force des études de Hergé, de Mœbius, les carnets de Druillet, les travaux de Franquin, de Fred, de Gotlib,

1. Sur le plan du volume des ventes, s'entend. Les «États généraux de la bande dessinée » ont récemment démontré la précarité des auteurs et souvent celle de leurs éditeurs.

2. Ce qui était un objet de salles de ventes est devenu aujourd'hui un produit vendu en ligne pour les collectionneurs du monde entier, dans des proportions qui dépassent complètement le marché de l'art ou du manuscrit d'auteur.

3. Voir sur ce point L. Vigier, «Génétique de la bande dessinée», Littérature, n 178, juin 2015, p. 80-92.

Le premier lieu génétique : l'album et ses marges 


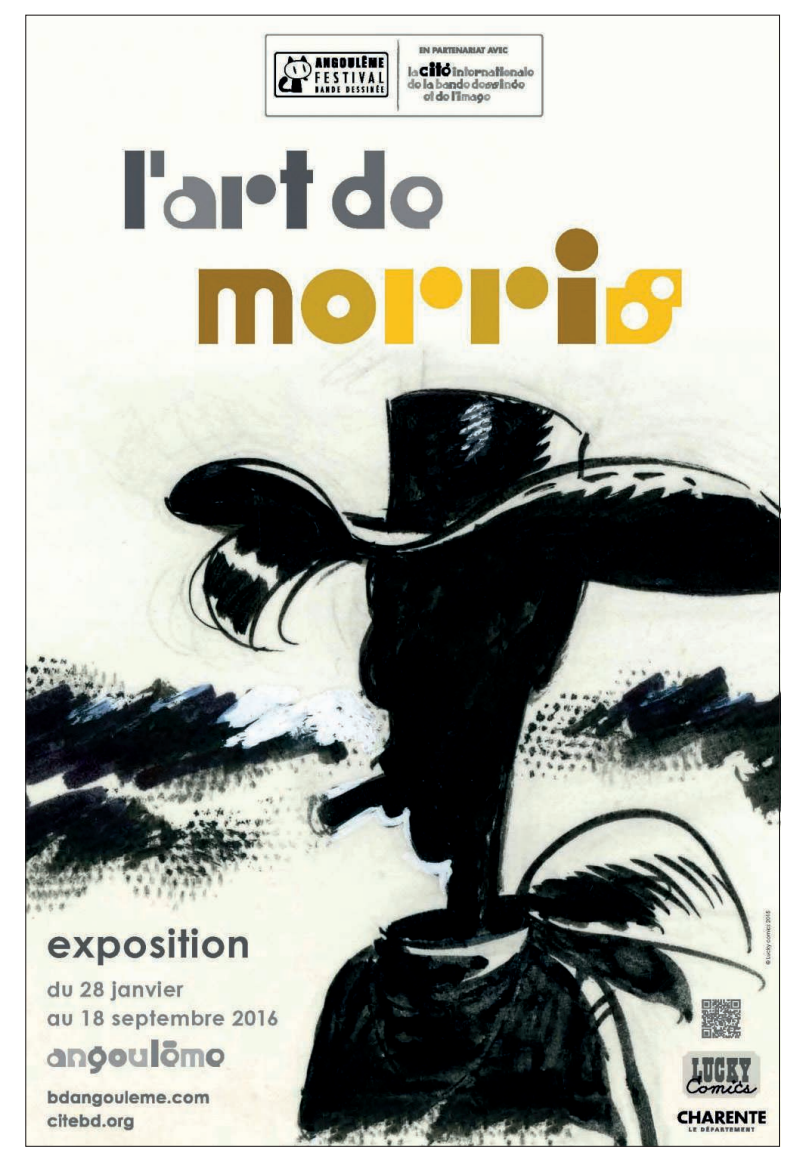

Fig. 1 : Affiche de l'exposition «L'art de Morris», Cité de la bande dessinée, Angoulême, Lucky Luke (C) Lucky Comics, 2016

\section{La séance de dédicace ou le sentiment des naissances}

qu'on avait imaginés, mais aussi le génie graphique de Greg, Peyo, Will, Morris ${ }^{4}$, qu'on ne soupçonnait pas.

On s'immerge, avec le regard d'un amateur d'art, dans la complexité des dessins préparatoires de Rochette pour le Transperceneige au musée Géo-Charles à Echirolles (fig. 2 et 3). Entre cette strate première de la composition et l'édition de l'album dans sa version définitive, un continent entier apparait, fait de recherches graphiques, de contraintes éditoriales, de trouvailles scénaristiques et formelles. On décrypte une histoire des origines, on se laisse prendre au spectacle et à la mise en scène des processus d'élaboration, à la fois instructifs et hypnotiques.

Cette fascination jamais démentie pour les dessins antérieurs à l'édition se repère assez aisément dans le succès des séances de dédicaces, après la publication, dont la nature est fort complexe. Bien plus riche qu' une signature (dont elle conserve la dimension auctoriale), elle vient imiter le brouillon, en rejoindre le statut et la notoriété et surtout en répliquer la fraîcheur d'original. La dédicace, en ce sens, joue et met en scène l'étape de la genèse et c'est un phénomène spécifique de la bande dessinée. S'il est fréquent que la demande se fasse au profit de personnages et de décors issus de l'univers de l'auteur, il s'agit aussi

4. En témoigne la récente exposition des dessins de Morris pour Lucky Luke au Musée de la Bande dessinée à Angoulême, «L'art de Morris », Festival d'Angoulême/Lucky Comics, 2016 (fig. 1 et 4). 

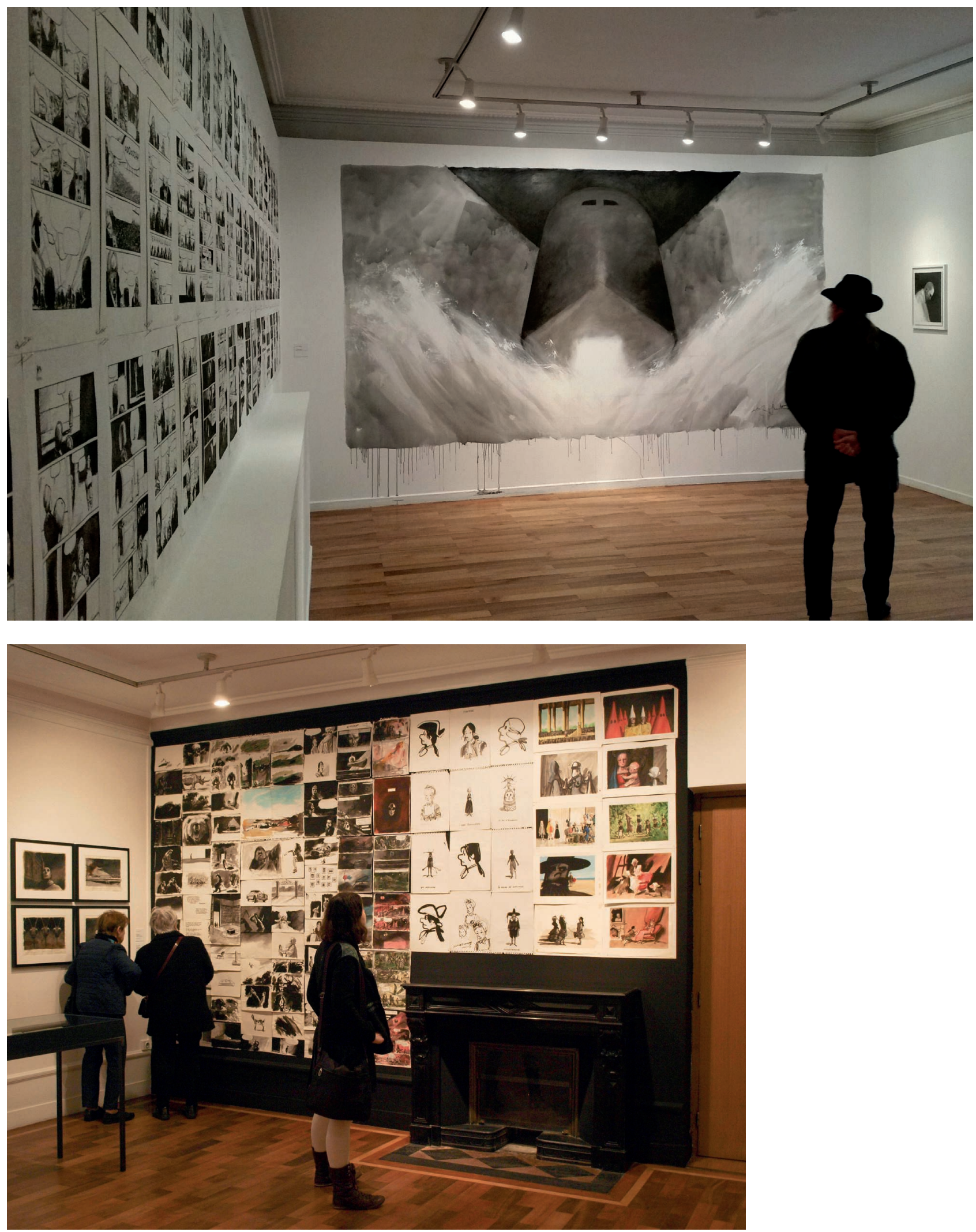

Fig. 2 et 3 : Exposition Jean-Marc Rochette, Le Transperceneige - Bandes dessinées, dessins et illustrations (Musée Géo-Charles-Echirolles, 20 février-17 mai 2015

Commissariat : Elisabeth Chambon, conservateur en chef du patrimoine du musée) Photo Manon Billard @ Jean-Marc Rochette 


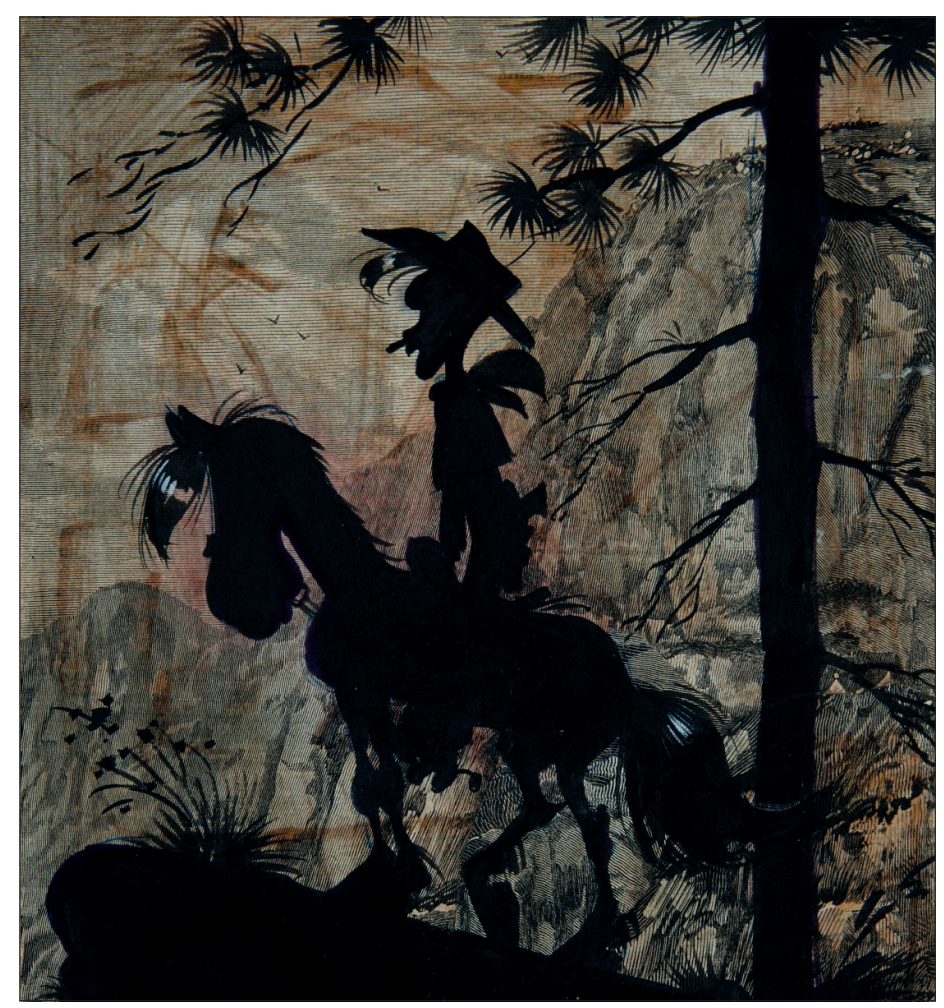

Fig. 4 : Lucky Luke sur Cheval en ombre chinoise (C) Lucky Comics, Festival d'Angoulême, 2016

pour celui qui vient demander cet autographe très spécial de s'insérer de manière furtive et a posteriori dans le travail de fabrication en sollicitant une nouvelle apparition visuelle du personnage de l'album. Les motivations de l'acheteur-prescripteur ne sont pas toutes artistiques : les dédicaces (qui sont d'ailleurs des envois) sont certes souvent affectives, narcissiques, évidemment fétichistes, et parfois directement commerciales. Mais reste qu'en posture d'attente et de respect, le prescripteur du dessin assiste en témoin hypnotisé à une naissance, au début de quelque chose de magique qui lui est adressé, à la fois suite et passé de l'œuvre. Du côté de l'auteur en état de promotion, c'est au mieux un dessin réussi en un temps record, et plus généralement une rencontre inachevée avec les lecteurs, plus souvent enfin un exercice qui relève du travail à la chaîne (fig. 5).

Les originaux après l'album
De leur côté, les planches originales, parfois même des cases uniques traitées comme tableaux, s'affichent aussi comme cuvres dans les expositions (souvent en marge des festivals mais de plus en plus dans les salles des musées et des expositions d'art contemporain, par ailleurs sur de très actifs sites de vente en ligne), où les étapes antérieures à l'impression atteignent des prix astronomiques proches des cotes de certains artistes contemporains, non sans confusion des territoires ${ }^{5}$. Cette valorisation spectaculaire de l'avant-imprimé vient contrer la valorisation de l'album original chez le collectionneur, sans l'annuler totalement, et a le mérite, sur le plan scientifique, de déplacer l'intérêt vers le processus artistique et technique situé en amont de l'édition.

5. La tradition ancienne en littérature du faux manuscrit (l'auteur recopiant telle page de roman ou tel poème pour le vendre) fonctionne à plein régime en bande dessinée, les auteurs tirant de ces productions des bénéfices non négligeables. 
Les allées du Festival d'Angoulême regorgent ainsi de planches de tout format qui atteignent des prix, pour les auteurs les plus connus, de plusieurs milliers d'euros par planche. Disposées en masse (certains stands affichent des centaines de références), les planches émettent des signaux variés : objets d'un marché et support de spéculation, elles sont aussi des appels de grande force à l'étude, à l'observation minutieuse et à la réflexion. À plus d'un titre, elles fonctionnent comme textes visuels et requièrent un déchiffrement spécial. C'est sur une partie de cet appel que repose la production de documentaires sur la fabrique de la bande dessinée, qui prend, depuis quelques années, la dimension paradoxale du teasing génétique.
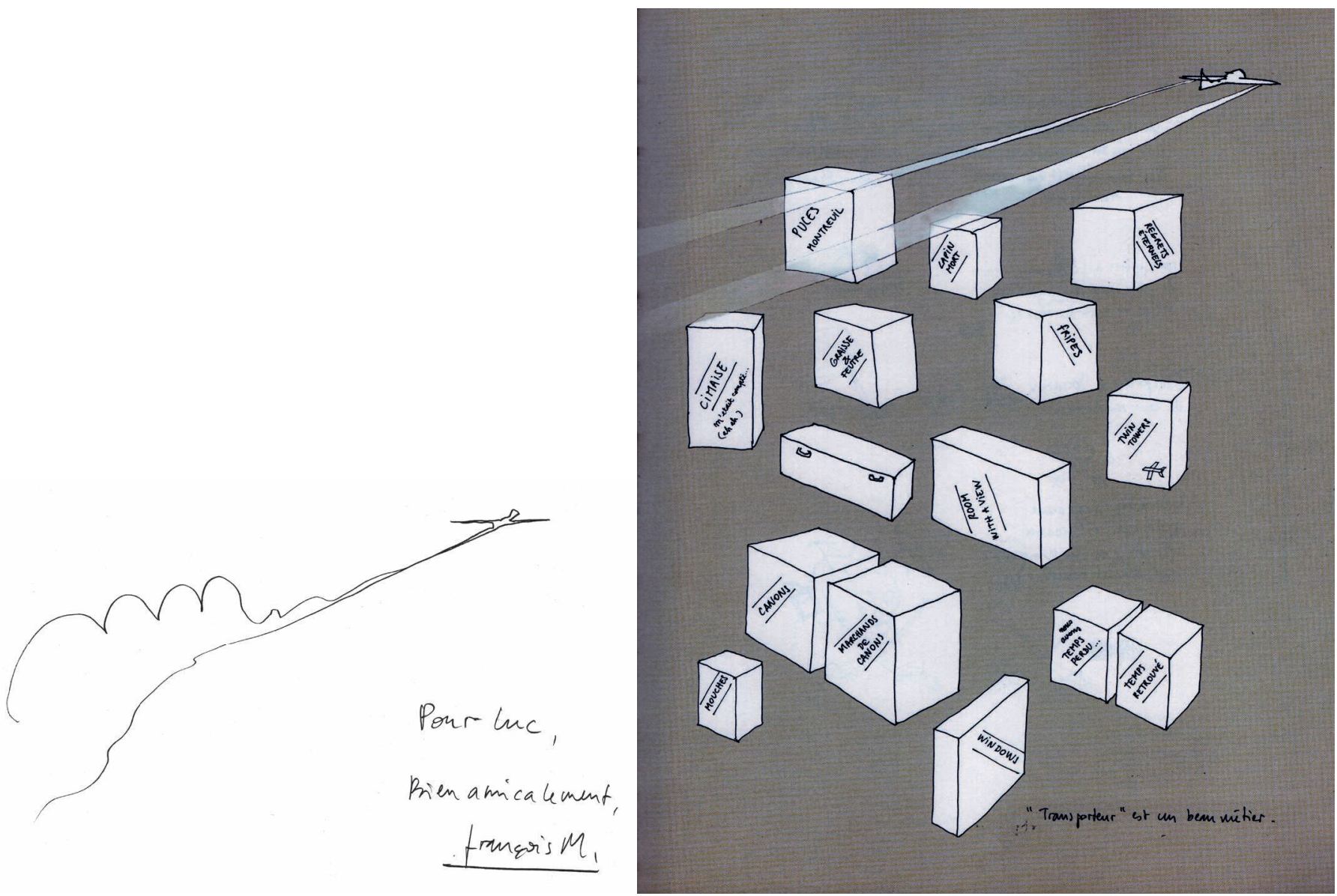

Fig. 5 : À gauche : François Matton, dédicace à l'auteur; à droite : François Matton, De pièces en pièces (P.O.L, 2007), détail de la page 9 
Génétique du teasing
La télévision, qui a rapidement compris l'intérêt visuel et la force d'attraction du dessin filmé auprès d'un public très large 6 , pèse depuis son émergence sur la vision graphique des dessinateurs et des premiers grands - qu' on a envie de nommer les pères de la bande dessinée. En France, ce sont les émissions de télévision qui ont d'abord vulgarisé, au milieu des années soixante-dix avec $\mathrm{Cabu}^{7}$, Greg, Druillet et bien d'autres, la pratique du dessin de caricature, du graph satirique, du cadavre exquis sur paper-board, ou simplement ludique, multipliant les séances pédagogiques et introduisant dans le territoire couvert par la télévision non l'art de la bande dessinée, mais quelques-uns de ses principes intellectuels et de ses étapes techniques. On mesure le chemin parcouru en songeant à l'excellente série Graph qu' avait lancée la chaîne franco-allemande Arte dans les années quatre-vingt-dix, où c'est le statut d'artiste qui était nettement soutenu, avec des scènes anthologiques où l'on avait pu voir Topor dessinant dans l'instant sa propre forme et délavant les fusains au vin blanc qu'il était en train de boire8.

Le modèle contemporain de marketing et de diffusion de la bande dessinée, lui-même influencé par les annonces des feuilletons à venir en littérature dans la presse ou dans les comics books, vient aujourd'hui contaminer les modalités de mise en valeur du travail d'artisan et d'artiste. Le cas des making-of, qu'on donne ordinairement après et en bonus dans les DVD d'animation ${ }^{9}$, reporte un instant l'attention vers ce qui précède chronologiquement la parution de l'album. Le site des Éditions Futuropolis, combinant la puissance de la vidéo et celle de la mise en ligne, met fortement l'accent sur le work in progress de ses auteurs et souligne un certain nombre des points de fabrique de l'image qui viennent à la fois conforter l'importance d'une génétique de la bande dessinée et banaliser en la publiant largement - et presque naturellement aujourd'hui - ce qui dans d'autres domaines relève de la découverte et de l'archéologie secrète.

L'exemple du film ${ }^{10}$ réalisé pour le travail de Manuele Fior, Les Variations d'Orsay ${ }^{11}$, en collaboration avec le musée d'Orsay (fig. 6), brasse des éléments fondamentaux : sous le titre «Trouver des formes», se déploie un discours pédagogique très précis, mixte du portrait d'auteur et de l'enquête sur une pratique artistique. Le film - non sans effets de mise en abyme médiatiques - s'amorce par un plan-séquence sur les originaux et dédicaces accrochés dans le couloir près du bureau du dessinateur, se poursuit par des plans du bureau-atelier modeste et resserré. Suit la présentation par l'auteur d'un projet qui justement se situe au croisement du patrimoine des arts visuels (musée d'Orsay) et des techniques de dessin, de narration et de mise en couleurs de la bande dessinée.

6. On pense notamment à l'émission Tac-au-Tac (ORTF, 1969-1975). Plus récemment, le développement des «concerts dessinés », où un groupe de musiciens joue sur scène pendant qu'un dessinateur réalise une performance graphique en direct, appartient à une histoire scénique complexe plus ancienne mais confirme la goût inaltérable du public pour la réalisation directe du dessin, qui confine à la magie retrouvée des tracés rupestres.

7. L'émotion suscitée par la mort atroce de Cabu le 7 janvier 2015 confirme la présence de cette incarnation du dessin satirique dans la mémoire collective de plusieurs générations.

8. Cette émission d'un format de treize minutes fait partie d'une série de quatre films de Marie-Dominique Dhelsing consacrés à Ralph Steadman, Ernest Pignon-Ernest, Roland Topor, François Schuiten; collection «Graph», Arte, 1992-1993.

9. On songe notamment à l'animation française, avec le remarquable making-of de Persepolis (Marjane Satrapi, Vincent Péronnaud, 2007) ou à celui de Bécassine de Philippe Vidal, 2002.

10. < https://vimeo.com/139057829>

11. Futoropolis, septembre 2015. 


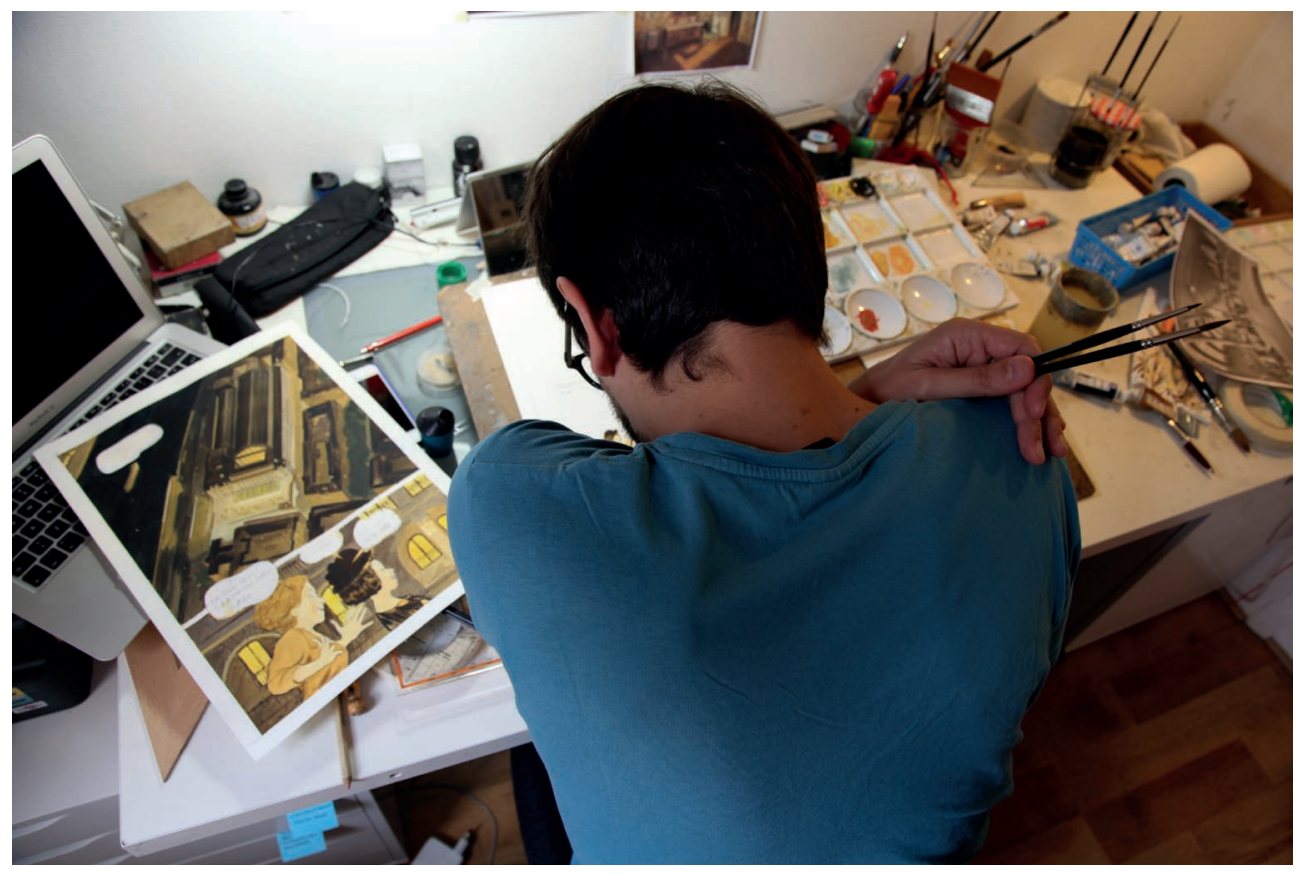

Fig. 6 : Photogramme extrait du reportage de Massimo Colella

(«Trouver des formes») pour les Éditions Futuropolis

(C) Massimo Colella, La Bande Destinée, 2015

Ce projet, dit l'auteur, «est comme une boîte de Pandore d'où j'extrais des suggestions graphiques et narratives reçues en visitant les salles du musée», ce qui implique une forme de hasard très minime dans le projet : il y a eu intention d'hybridation esthétique grande peinture/technique de la bande dessinée mais également une recherche intense de formes à l'intérieur de cette genèse téléguidée, où peut aisément se loger une démarche qui intéresse la génétique des œuvres, pour peu que l'auteur et l'éditeur aient conservé de ces moments des traces tangibles. En l'occurrence, elles sont relativement maigres : l'auteur feuillette des planches presque achevées, sur lesquelles les bulles restent vides et aucune trace des processus d'élaboration n'est visible. En revanche, et c'est une dimension que soulignent bien Jean-Pierre Mercier et Benoît Peeters dans ce numéro de Genesis, la donnée technique devient première et pourrait bien prendre une dimension inédite pour créer un espace de recherche qui serait celui d'une génétique polymorphe impliquant la prise en compte de la succession des choix d'outils et de supports.

Ce dont parle Manuele Fior, ce n'est en effet pas d'abord des processus antérieurs de création, mais des modalités techniques de réalisation, qui elles aussi ont une histoire, dans la mesure où l'éditeur souhaite ici représenter, évoquer et suggérer la peinture dans la bande dessinée. Or c'est aux techniques picturales justement qu'a recours cet auteur et c'est pour sa maîtrise des à-plats de couleurs à la gouache que le dessinateur a été choisi par l'éditeur. On serait donc devant des processus orientés, comme on a pu en rencontrer au XIXe siècle 
dans les écritures verniennes ${ }^{12}$, conçues d'avance, rédigé à deux ou quatre mains, corrigées par l'éditeur en fonction du concept initial et conforme au plaisir attendu de la lecture.

Que reste-t-il d'une génétique de la bande dessinée dans ce cas ? Manuele Fior fait à cette question une réponse précise : « Le travail du dessinateur est aussi un travail d'études », «études» pris au sens des écoles d'art visuels et plastiques où la recherche d'un style sur modèle et sous contrainte fait justement partie du travail de fond des artistes. Ce qui impliquerait une génétique des éléments de recherche et des études préparatoires. Le reportage ne manque pas de pointer vers des processus qui sont spécifiques à ce type de démarche et de recherche, impliquant aussi l'influence déterminante de maîtres en bande dessinée, Manuele Fior signalant que Mattoti lui a conseillé de ne pas changer de technique à chaque album et d'approfondir celle qu'il possède le mieux. Le mur du dessinateur, un instant capté par la caméra, est porteur d'indices utiles : dans un panneau situé au-dessus de sa table se mélangent des dessins qui lui appartiennent et des dessins qui sont les œuvres d'autres artistes, où l'auteur dit chercher les bifurcations d'idées graphiques les plus stimulantes.

Au centre des processus ici décrits, l'élément central de l'inter-iconicité se présente comme si complexe qu'il est susceptible de fournir à lui seul un matériau gigantesque à l'approche génétique : proximité et détachement du référent, sémantique du palimpseste, jeu des citations totales ou partielles, citations par la couleur et évolution progressive du degré d'implication et d'éloignement de l'auteur, problèmes techniques, contournements, solutions.

Ainsi filmé, ce qui se passe est tout simplement de l'ordre de la magie qui nous lie au geste parfait de l'artiste, de l'artisan, du chorégraphe, à ceci près qu'on peut détecter dans la résistance technique les éléments furtifs d'une décision graphique différente. Mais l'essentiel demeure masqué : dans les paroles prononcées par le dessinateur dans ce reportage, où une forme de spontanéité et de concentration semble avoir été saisie, ce qui frappe, c'est que le moment de l'exécution, de la réalisation de la planche reste certes le plus spectaculaire mais le moins génétique qui soit. C'est le travail de fond auquel l'artiste renvoie qui fait le matériau: l'étude longue et ancienne des lumières, les recherches sur le new style, les textiles, les lieux, les personnages, les vêtements, les atmosphères d'époque.

\section{Numérisation mon amour}

La vidéo en ligne n'est pas le seul médium de cette magie d'atelier. Outre les premières créations de bandes dessinées électroniques et interactives natives (de Witches and Stitches de 1985 à Argon Zark en 1995), qui font entrer les comics dans l'ère vidéo-ludique des supports électroniques, c'est la numérisation des planches qui, précisément sur cette période, donne une dimension nouvelle à la diffusion des albums, de leurs extraits et de leurs versions d'étape, créant une nouvelle nature de l'image. Un dessin numérisé change en effet d'espace, de texture, d'être ${ }^{13}$. Pris dans la nappe du dispositif de projection qu'est l'écran, la lumière du rétro-éclairage (annulée seulement dans les modèles les plus austères de liseuses) traverse le trait, change les couleurs - parfois même les perspectives les plus rigoureuses - et génère

12. Voir Philippe Scheinhardt, Jules Verne : un processus d'écriture sous contraintes, Genesis, n 33, 2011, p. 173-186.

13. On lira à ce sujet le très stimulant L'Être et l'écran de Stéphane Vial (Paris, Presses universitaires de France, 2013). 
une existence nouvelle du dessin, qui en retour modifie sa perception par l'auteur et ses travaux ultérieurs : unification et adoucissement ou au contraire effet de texture et de crénelage, modification des échelles, agrandissements démesurés, la numérisation crée une seconde couche de manifestation graphique de l'image, qui n'a rien à voir avec l'original et qui souvent magnifie d'autres types de phénomènes graphiques : la couleur, le dynamisme, la perspective, l'existence visuelle de l'étude, de nouveaux formats et de nouveaux effets de cadre apparaissent.

L'écart bien évidemment se resserre avec le dessin numérique direct, déjà très ancien dans les pratiques graphiques (Mœbius s'y était intéressé dès le début des années quatre-vingt-dix), qui exploite un effet inverse de plus en plus saisissant : celui d'une identité parfaite entre le tracé et son rendu numérique, la possibilité d'exploiter des espaces complètement hors norme, panoramas gigantesques, suivi narratif horizontal sur la totalité du strip, verticales vertigineuses des scroll comics. Dans la même logique, le dessin assisté par logiciel - on lira dans ce numéro le témoignage d'Alexandre Clérisse sur Illustrator -, en éliminant un certain nombre d'étapes sur papier, change totalement la donne graphique en s'installant dans un matériau numérique de manière native et non par transposition. D'abord pris en charge par les éditeurs, les maquettistes et les imprimeurs, les versions scannées des planches sont devenues progressivement des matières premières de l'étude graphique avant de devenir des éléments naturels de la médiatisation de la bande dessinée et de l'exposition de ses processus au sein du web, où se croisent les travaux papiers numérisés, les tracés numériques et les productions assistées par ordinateur. De ce point de vue, les nouveaux territoires numériques de la bande dessinée sont des lieux observables de nouvelles méthodes de composition et de recherche de formes.

Les archives de l'Internet nous rappellent que le premier temps fut celui, peu dynamique mais déjà étonnant, des sites statiques où l'on vit apparaître les planches numérisées et les travaux amateurs. Les premiers blogs BD, aux frontières des années deux mille et au début $\mathrm{du}$ «web 2.0 », font entrer dans la boucle les avis, les adorations, les gloires éphémères ou pérennes, où l'on devine aussi une féminisation de plus en plus nette de la profession : des dizaines d'autres jeunes auteurs mettent en ligne leurs esquisses, leurs carnets, leurs techniques, leurs projets, passant parfois à l'édition papier de leurs «notes». En France, les années 2000-2010 sont d'une créativité impressionnante : des dizaines d'artistes y font leurs armes et leur école, soit en parallèle d'un métier déjà rémunérateur (rare), soit sans aucune expérience professionnelle, avec un impact considérable sur les communautés de fans. Leur curiosité déclenche souvent, de la part de l'auteur du blog, la création de tutoriels techniques ou d'historiques vidéo qui peuvent aller du simple conseil à la performance éblouissante. Souvent, ces étapes sont elles-mêmes connectées aux dossiers préparatoires et techniques demandés par les éditeurs (gammes et roughs de personnage, recherches de costume, recherches de décor, essais d'ambiances, éléments qui mériteraient en eux-mêmes une étude, tant sont identifiables les attentes commerciales, la présence d'un design par cible de lecteur, mais aussi les influences esthétiques et les écoles). Plus récemment, le making-of vidéo accéléré d'un dessin particulièrement travaillé est devenu une sorte de genre international présent sur les blogs et les réseaux de vidéo en ligne, où la virtuosité technique continue d'exercer sa force de séduction (fig. 7). 


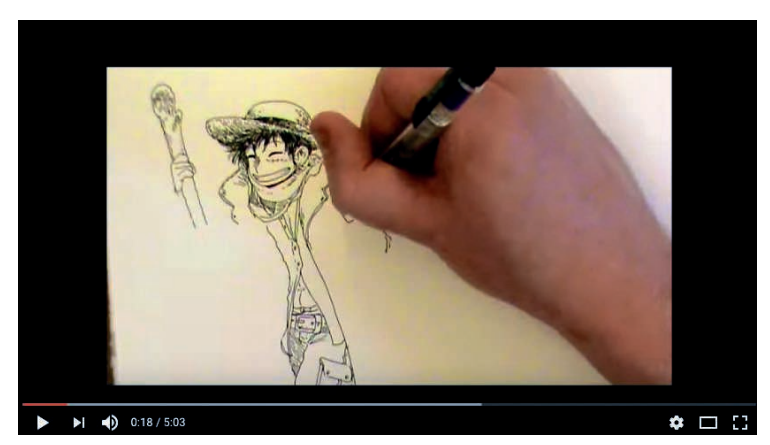

Fig. 7 : Boulet, Hommage au manga One Piece, 2012.

Temps réel : 5 heures (capture d'écran)

(www.youtube.com/watch?v=fWaTn71JLNc)

\section{Carnets d'esquisse et réseaux d'images}

Les auteurs familiers du net sont en effet les premiers à attirer notre attention sur la nouvelle forme que prend leur carnet, leur atelier, leur manière de travailler. On trouve en ligne la numérisation de nombreuses pages mais également des travaux numériques tracés sur tablette numérique ou sous logiciel vectoriel. En complément du site officiel ou du blog, les éléments de génétique se dispersent sur d'autres supports, notamment les réseaux sociaux fondés sur le partage d'images, que fréquentent abondamment amateurs et professionnels : Facebook, mais également Tumblr. et Instagram, font partie de ces réseaux où l'étude sur carnet trouve naturellement à s'exposer, soit de manière très anarchique, soit de manière plus organisée et volontaire, l'archive changeant parfois de statut pour devenir, comme chez Sfar, billet d'humeur et histoire dessinée de soi.

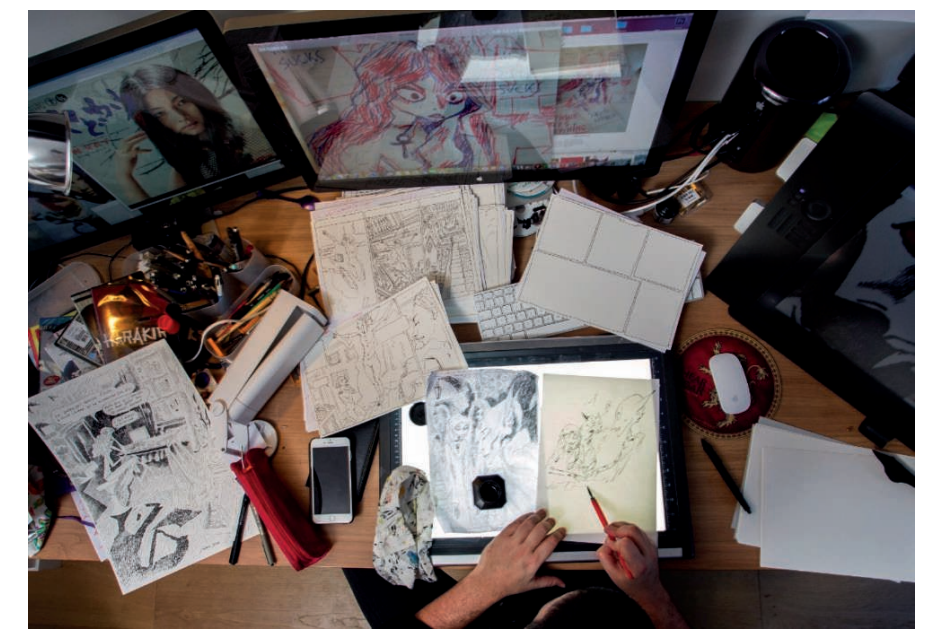

Fig. 8 : Joann Sfar à son atelier, page Facebook de l'auteur (C) Joann Sfar 
La page Facebook de Joann Sfar (fig. 8) est emblématique de cette nouvelle exhibition des procédures : non seulement on aperçoit des esquisses (pour Le Chat du Rabbin 14) et on voit des dessins produits au jour le jour, mais on peut également collecter des informations génétiques volontiers dispensées. Un petit film mis en ligne en février 2016 permet ainsi d'entendre l'auteur s'expliquer sur le croisement des contraintes de lisibilité de l'image pour un format A3 exécuté au pinceau et des contraintes éditoriales imposées par le souhait d'efficacité de Paris Match. La spécificité du discours ici est que la génétique de la bande dessinée ne peut se dire sans passer par les enjeux du support, de l'outil - en l'occurrence le pinceau, qui devait faire du noir mais qui, insuffisamment rincé, a produit des rehauts de bleu -, du format et du temps. La tension entre ce que Sfar nomme le «dessin longuement préparé» et le dessin spontané, composé au dernier moment, correspond ici également au contexte de la presse et de ses urgences. Dans ce cas comme dans bien d'autres, l'interface numérique fonctionne comme surface auto-éditoriale d'une génétique qui ne peut être que partiellement exposée.

La fluidité technique des sites web et la force visuelle des éléments graphiques numérisés ont favorisé le développement d'approches paragénétiques, qui existaient déjà dans les revues spécialisées depuis les années quatre-vingt. Les travaux des Cahiers de la bande dessinée, par exemple, où l'on pouvait trouver une rubrique consacrée à l'étude des planches et à l'explication de leurs processus de fabrication et de fréquentes couvertures représentant «l'artiste en son atelier ${ }^{15}$ », se sont prolongés dans la revue $9 e$ Art ${ }^{16}$, dont la version web plonge volontiers le regard au cœur des processus créatifs. La rubrique «Dans l'atelier...» est particulièrement étoffée (fig. 9). Sans nécessairement insérer des études ou des carnets, cette rubrique met l'accent sur l'auteur, sur son travail de réflexion, ses choix techniques, ses évolutions. Par ailleurs, les entretiens révèlent fréquemment une utilisation plus ou moins régulière, par les auteurs, des sites web et des blogs, où s'affichent leurs travaux en cours. L'approche par les coulisses crée ici, au sein du médium numérique, un espace où les éléments graphiques prennent le statut d'objet d'étude mais, comme on l'a vu plus haut, d'objets accentués par la lumière de l'écran, situés dans un autre dispositif visuel, lui-même placé dans une constellation de sites liés entre eux. Si une génétique de la bande dessinée veut prendre en compte la dimension numérique, elle doit alors tenir ensemble les objets classiques constitués par la time-line des carnets dans leur ordre et les liaisons multiples en réseau générant des influences croisées au sein d'une société de dessinateurs qui dispose aujourd'hui, grâce au web, d'un panoptique numérique considérable, générant de nombreuses interactions, citations et influences.

14. Joann Sfar et Brigitte Findakly (couleurs), Le Chat du Rabbin, Paris, Dargaud, six albums (2002-2015).

15. On notera dans ces numéros très riches des années soixante-dix (le n 24 du deuxième trimestre 1974 consacré à Claire Brétecher et le $n^{\circ} 38$ du troisième trimestre 1978 consacré à Alexis, par exemple) la rareté des brouillons, qui seront davantage présents dans les numéros des années quatre-vingt.

16. Revue dirigée par T. Groensteen, aujourd'hui en ligne; quinze numéros papier parus depuis 1996, en ligne uniquement depuis 2010 .

\section{Les approches externes}




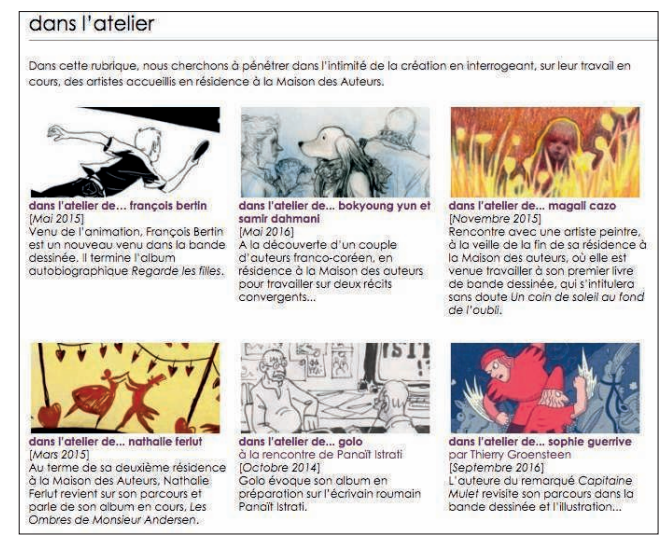

Fig. 9 : Capture d'écran du site de la revue $9 e$ Art (http://neuviemeart.citebd.org/spip.php?rubrique112)

\section{Panoptique paradoxal}

Il n'est donc pas certain que ce panoptique spectaculaire du dessin abrité par le réseau, sans doute très efficace sur le plan de la séduction, soit un terrain favorable à une étude méthodique des sentiers de la création en bande dessinée : il pourrait même entraver l'idée d'un parallèle possible ou attendu avec la génétique des œuvres littéraires, en ce qu'il éteint la singularité même du processus créatif. Simultanément, s'il fonctionne comme un leurre, il n'en contient pas moins des indices furtifs qui pointent vers des pratiques plus approfondies et des dossiers génétiques plus anciens. C'est ce que montrent, à travers leurs carnets, dans ce numéro de Genesis, les approches autogénétiques de nos auteurs.

L'exhibition vidéo-numérique des coulisses de la bande dessinée, comme ses modes d'exposition plus anciens, offrent donc un paysage riche, mais délicat à appréhender. Elle produit des données exploitables en grand nombre, depuis les documents classiques sur papier aux formes nouvelles, qui constituent à l'évidence un appel à l'étude et à l'archivage systématique. Par ailleurs, le phénomène des interactions graphiques nées des travaux d'atelier, les observations à distance des pratiques, les hommages croisés rendus d'un auteur à l'autre générant une sociologie génétique, l'autodidaxie latente ou affichée des auteurs entrent dans l'étude des processus de fabrication. Le réseau des dessinateurs fonctionnerait ainsi en disposant sous nos yeux les œuvres autrefois invisibles ou inaccessibles issues des ateliers.

Plus largement, et de manière nouvelle, le réseau dirait, à sa manière anarchique et proliférante, les possibilités d'hybridations stylistiques, d'inspirations superposées, désignant indirectement le web comme l'une des matrices possibles de leurs créations : sources photographiques, graphiques, visuelles, sonores (la musique joue un rôle considérable dans la mise en scène visuelle de leurs travaux chez certains dessinateurs), le réseau se superpose au réel comme lieu d'observation, de mémoire, d'enquête, et une génétique de la bande dessinée, qui doit s'établir sur les archives classiques, peut aussi s'étendre à une génétique élargie des manifestations médiatiques de la bande dessinée, qui combine l'origine, la fabrication et la réception de l'image. 
LUC VIGIER est maître de conférences à l'université de Poitiers et dirige l'équipe Aragon de l'ITEM depuis 2008. Il est également responsable du Master Bande dessinée à l'École européenne supérieure de l'image à Angoulême. Ses travaux portent principalement sur l'œuvre en prose d'Aragon, la question du témoignage au XXe siècle, le mouvement Dada, le surréalisme, les musées imaginaires, le dessin des écrivains et la bande dessinée, enfin l'image numérique des textes littéraires. Il a publié Le Paysan de Paris (édition commentée, 2004) et Le Surréalisme (2006) en Bibliothèque Gallimard et coordonné des colloques sur Aragon («Aragon politique» en 2008 et «Aragon et Les Lettres françaises» en 2013 aux Presses universitaires de Strasbourg). Il a publié plus récemment une étude des jeux de coécriture entre Aragon et Breton dans le numéro 41 de la revue Genesis ( Créer à plusieurs mains », 2015) et Aragon et le cinéma (2015) aux Nouvelles Éditions Jean-Michel Place.

luc.vigier@univ-poitiers.fr

Résumés

\section{Bande dessinée : une génétique à ciel ouvert}

Cette étude générale s'intéresse moins aux dossiers génétiques eux-mêmes qu'à leur médiatisation via les bonus, suppléments et autres making-of, présents non seulement dans les albums mais depuis quelques années sur la Toile et au cœur des réseaux sociaux, où se combinent les vidéos d'artistes au travail, les reportages sur la fabrication des albums, les reproductions de carnets d'études, les études critiques. On interroge les connexions problématiques de ces différents espaces d'exposition du processus graphique qui relève à la fois de l'archéologie et du spectacle.

This general study deals less with genetic dossiers as such than with their media coverage through bonuses, supplements and other making-of, present not only in albums but for the past few years on the Net and on social networks where videos of artists at work, reportages on the making of albums, reproductions of sketchbooks, critical studies are combined. We will examine the problematical connection of the various exhibition spaces of the graphic process that belongs both to archeology and performance.

Diese allgemeine Studie beschäftigt sich weniger mit genetischen Dossiers selbst als mit ihrer Mediatisierung via Bonusmaterial, Supplementen und anderen Making-of, die nicht nur in den Alben zu finden sind, sondern seit einigen Jahren auch im Netz und im Kern der sozialen Netzwerke, wo sich sowohl Videos von Künstlern bei der Arbeit als auch Reportagen über die Herstellung von Alben, Reproduktionen von Skizzenheften und kritische Studien finden. Wir untersuchen die problematischen Verbindungen zwischen diesen verschiedenen Räumen der Darstellung des graphischen Prozesses, der zugleich an Archäologie wie auch an Performance erinnert.
Este estudio general se interesa menos por los dosieres genéticos que por su mediatización a través de los suplementos, extras y otros making-of, presentes no solamente en los libros, sino también, desde hace algunos años, en la web y en el corazón de las redes sociales, donde se combinan los videos de artistas en plena actividad, reportajes sobre la fabricación de las historietas, reproducciones de libretas de estudios, análisis críticos. Se observan aquí las conexiones problemáticas de esos diversos espacios de exposición del proceso gráfico que dan cuenta tanto de la arqueología como del espectáculo.

Este estudo ocupa-se menos menos de dossiers genéticos que da mediatização exercida por documentários, suplementos e outros tipos de making-of, presentes não só nos álbuns, mas ultimamente na internet e nas redes sociais, onde se combinam vídeos dos artistas em atuação, reportagens sobre a fabricação dos álbuns, reproduções de blocos de notas e estudos críticos. Em questionamento estão as conexões problemáticas desses diferentes espaços de exposição do processo gráfico, que tanto têm a ver com a arqueologia como com o espetáculo.

Il seguente studio generale s'interessa meno ai dossier genetici che alla loro mediatizzazione tramite i bonus, i supplementi e altri making-of presenti non soltanto negli albi ma, da qualche anno, sul Web e sui social network, luoghi dove coesistono i video di artisti al lavoro, i reportage sulla produzione degli albi, le copie dei taccuini di studio, gli studi critici. Vengono interrogate le connessioni problematiche di questi differenti spazi di esposizione del processo grafico che pertiene ad entrambi i campi dell'archeologia e dello spettacolo. 